\title{
Simulation of the Magnetic Hysteresis Loop in Ferrimagnetism
}

\author{
Morishige Yoneda ${ }^{1, *}$, Shuji Obata ${ }^{2}$ and Masaaki Niwa ${ }^{3}$ \\ ${ }^{1}$ Japan Electronics College, Tokyo 169-8522, Japan \\ ${ }^{2}$ School of Science \& Engineering, Tokyo Denki University, Hikigun, Saitama 350-0394, Japan \\ ${ }^{3}$ School of Engineering, Tokyo Denki University, Tokyo 120-8551, Japan
}

Investigated the nano-scale $\mathrm{Fe}_{3} \mathrm{O}_{4}$ which is ferrimagnetic material that has attracted attention as an application of the spintronics. In order to investigate the magnetization process of $\mathrm{Fe}_{3} \mathrm{O}_{4}$ of ferrimagnetism, we report the results that derive the magnetic hysteresis loop with various parameter by the computer simulation using the retarded trace method. [doi:10.2320/matertrans.MAW201502]

(Received April 15, 2015; Accepted May 7, 2015; Published June 19, 2015)

Keywords: $\mathrm{Fe}_{3} \mathrm{O}_{4}$, ferrimagnetic, ferrimagnetic material, magnetic hysteresis loop, computer simulation, retarded trace method

\section{Introduction}

Recently, for an application material of spintronics, the magnetization process in spin valve of $\mathrm{Fe}_{3} \mathrm{O}_{4}$ has been attracting attention. Since the spin valve ${ }^{1-3)}$ by $\mathrm{Fe}_{3} \mathrm{O}_{4}$ has a complex structure, essential understanding of the magnetization process is not sufficient. In this paper, our aim is to build a theory of magnetization process for spin valve of $\mathrm{Fe}_{3} \mathrm{O}_{4}$ as a ferrimagnetic material and derive the magnetic hysteresis loop with various parameter by the computer simulation using retarded trace method. ${ }^{4-7)}$ This paper is composed as follows. In the next section, shows a magnetically ordered structure and crystal structure of $\mathrm{Fe}_{3} \mathrm{O}_{4}$ as a ferrimagnetic material, and explain about our model Hamiltonian. In section 3 , we describe the algorithm by the retarded trace method, and the simulation results. In section 4, as summary and discussion, we make a comparison between the our simulation results and some experimental results.

\section{The Model Hamiltonian in $\mathrm{Fe}_{3} \mathrm{O}_{4}$ as a Ferrimagnetic Material Model}

As shown in (a) and (b) of Fig. 1, $\mathrm{Fe}_{3} \mathrm{O}_{4}$ have a reverse spinel structure. These are made from octahedral site (B sites: $\mathrm{Fe}$ ion is surrounded by six oxygens) and tetrahedral site (A sites: Fe ion is surrounded by four oxygens). As shown in (c) of Fig. 1, the coupling between the A-site and B-site are antiferromagnet, the coupling at B-B or A-A are ferromagnet. As the starting point of the phase transition of complex systems that shown in the example of magnetite, since we are interested in only to the magnetic order in the present study, we introduced the Hamiltonian as a ferrimagnetic model as follows:

$$
H=H_{\mathrm{A}}+H_{\mathrm{B}}+H_{\mathrm{AB}}+H_{\mathrm{Zee}},
$$

where

$$
H_{\mathrm{A}} \equiv-\sum_{\langle i, j\rangle} J_{\mathrm{A}} S_{\mathrm{A}}(i) S_{\mathrm{A}}(j) \text {, }
$$

$-\sum_{\langle i, j\rangle} J_{\mathrm{B}} S_{\mathrm{B}}(i) S_{\mathrm{B}}(j), \quad H_{\mathrm{AB}} \equiv \sum_{i, j} J_{\mathrm{AB}}\left[S_{\mathrm{A}}(i) S_{\mathrm{B}}(j)+\right.$ $\left.S_{\mathrm{B}}(i) S_{\mathrm{A}}(j)\right]$ and $H_{\text {Zee }} \equiv \sum_{i}\left[S_{\mathrm{A}}(i)+S_{\mathrm{B}}(i)\right] h(i)$ are represent exchange interaction energy in A site, exchange interaction energy in $\mathrm{B}$ site, exchange interaction in between $\mathrm{A}$ and $\mathrm{B}$ site and Zeeman energy respectively. Here $J_{\mathrm{A}}>0, J_{\mathrm{B}}>0$

*Corresponding author, E-mail: yoneda@jec.ac.jp and $J_{\mathrm{AB}}>0$ are represent exchange interaction in $\mathrm{A}$ site, exchange interaction in $\mathrm{B}$ site, exchange interaction in between $\mathrm{A}$ and $\mathrm{B}$ site, respectively. Additionally $S_{\mathrm{A}}(i)$, $S_{\mathrm{B}}(i)$ and $h(i) \equiv \mu_{\mathrm{B}} H(i)$ ( $\mathrm{g}$ and $\boldsymbol{\mu}_{\mathrm{B}}$ are g-factor and Bohr magneton respectively) are represent spins of $i$-th lattice in A site, spins of $i$-th lattice in B site and applied magnetic field of $i$-th lattice respectively.

In this system, the free energy density $g(\beta, h)$ of the mean field approximation is given as follows:

$$
\begin{array}{r}
g(\beta, h)=\frac{z_{\mathrm{A}} J_{\mathrm{AA}}}{2} m_{\mathrm{A}}^{2}+\frac{z_{\mathrm{B}} J_{\mathrm{BB}}}{2} m_{\mathrm{B}}^{2}-z_{\mathrm{AB}} J_{\mathrm{AB}} m_{\mathrm{A}} m_{\mathrm{B}} \\
-\frac{1}{\beta} \ln \left\{\sinh \left[\left(2 S^{\mathrm{A}}+1\right) \beta \bar{h}_{\mathrm{eff}}^{\mathrm{A}} / 2\right] / \sinh \left(\beta \bar{h}_{\mathrm{eff}}^{\mathrm{A}} / 2\right)\right\} \\
-\frac{1}{\beta} \ln \left\{\sinh \left[\left(2 S^{\mathrm{B}}+1\right) \beta \bar{h}_{\mathrm{eff}}^{\mathrm{B}} / 2\right] / \sinh \left(\beta \bar{h}_{\mathrm{eff}}^{\mathrm{B}} / 2\right)\right\} .
\end{array}
$$

where $m_{\mathrm{A}} \equiv\left\langle S_{\mathrm{A}}(i)\right\rangle, m_{\mathrm{B}} \equiv\left\langle S_{\mathrm{B}}(i)\right\rangle, z_{\mathrm{A}}, z_{\mathrm{B}}, z_{\mathrm{AB}}, S_{\mathrm{A}}, S_{\mathrm{B}}$ and $\beta \equiv 1 / k_{\mathrm{B}} T$ are mean field of spins $S_{\mathrm{A}}(i)$, mean field of spins $S_{\mathrm{B}}(i)$, number of nearest neighbours in A site, number of nearest neighbours in $\mathrm{B}$ site, number of nearest neighbours in between A and B site, spin size in A site, spin size in B site and inverse temperature respectively. In addition, $\bar{h}_{\mathrm{eff}}^{\mathrm{A}}=$ $h+z_{\mathrm{A}} J_{\mathrm{AA}} m_{\mathrm{A}}-z_{\mathrm{AB}} J_{\mathrm{AB}} m_{\mathrm{B}}$ and $\bar{h}_{\mathrm{eff}}^{\mathrm{B}}=h+z_{\mathrm{B}} J_{\mathrm{BB}} m_{\mathrm{B}}-$ $z_{\mathrm{BA}} J_{\mathrm{BA}} m_{\mathrm{A}}$ are effective magnetic field in A site and $\mathrm{B}$ site respectively. The total magnetization $M(\beta, h)$ in ferrimagnet is then given by

$$
\begin{aligned}
M(\beta, h) & \equiv N g \mu_{\mathrm{B}} m(\beta, h) \\
& \equiv-N g \mu_{\mathrm{B}} \frac{\partial f(\beta, h)}{\partial h} \\
& =N g \mu_{\mathrm{B}}\left[S^{\mathrm{A}} B_{\mathrm{S}^{\mathrm{A}}}\left(\beta \bar{h}_{\mathrm{eff}^{\mathrm{A}}}{ }^{\mathrm{A}}\right)+S^{\mathrm{B}} B_{\mathrm{S}^{\mathrm{B}}}\left(\beta \bar{h}_{\mathrm{eff}^{\mathrm{B}}}^{\mathrm{B}}\right)\right],
\end{aligned}
$$

where $m(\beta, h)$ and $B_{\mathrm{S}}(x)$ are local magnetization in ferrimagnet and the Brillouin function respectively. In the next chapter, the free energy density $g(\beta, h)$ and the magnetization $m(\beta, h)$ are the starting point of our analysis method for Simulation of the magnetic hysteresis loop in ferrimagnetism.

\section{Basic Theory of the Retarded Trace Method}

In this section, in order to study the magnetic hysteresis loop of $\mathrm{Fe}_{3} \mathrm{O}_{4}$ by numerical simulations, we consider magnetization process from two different approaches. In first 

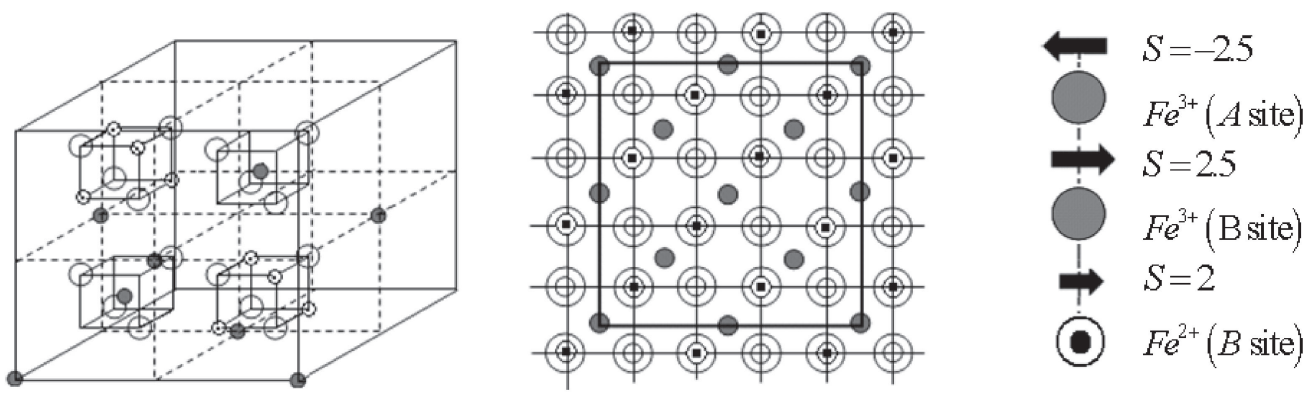

$A$ site $B$ site

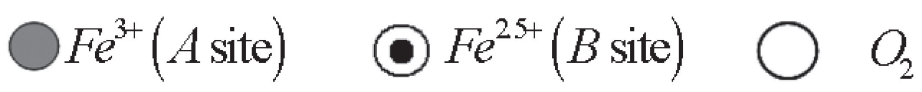

Fig. 1 Crystal structure, valence number of the electron charge and spin state in $\mathrm{Fe}_{3} \mathrm{O}_{4}$.

(a)

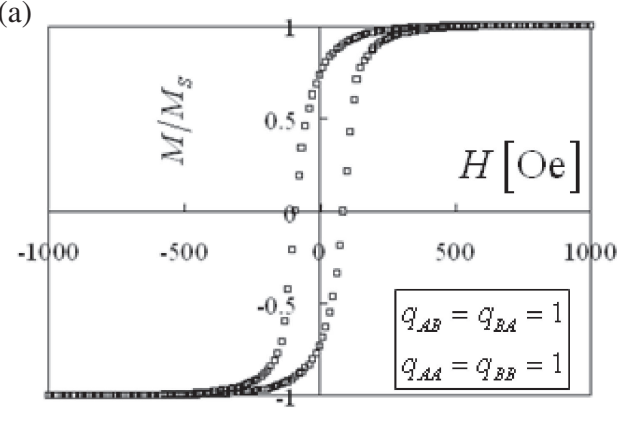

(c)

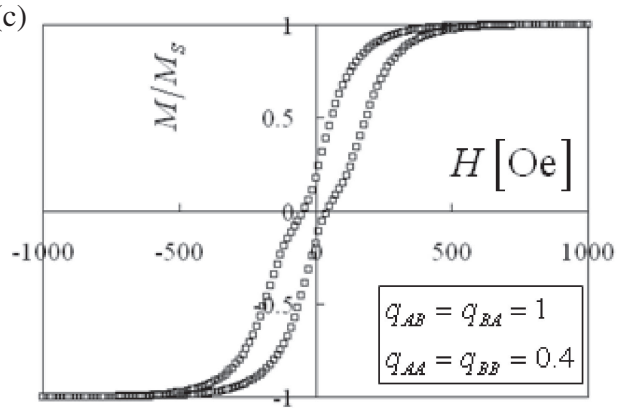

(b)

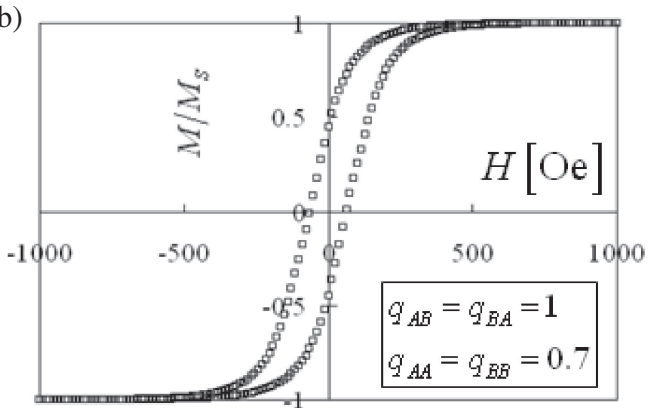

(d)

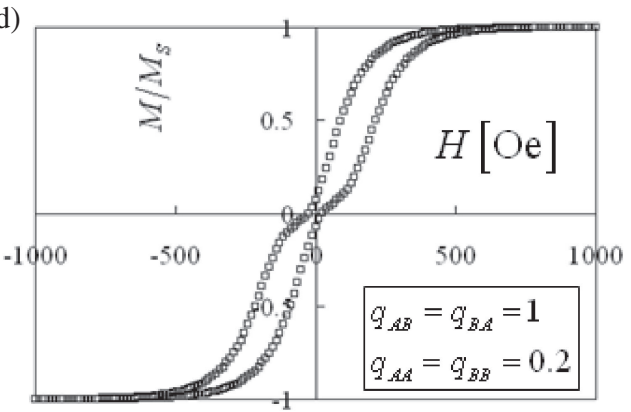

Fig. 2 The various parameters dependent magnetic hysteresis loops of $\mathrm{Fe}_{3} \mathrm{O}_{4}$ in temperature $T=300$ [K]. The value of the parameter are (a) $q_{\mathrm{AB}}=q_{\mathrm{BA}}=1$ and $q_{\mathrm{AA}}=q_{\mathrm{BB}}=1$, (b) $q_{\mathrm{AB}}=q_{\mathrm{BA}}=1$ and $q_{\mathrm{AA}}=q_{\mathrm{BB}}=0.7$, (c) $q_{\mathrm{AB}}=q_{\mathrm{BA}}=1$ and $q_{\mathrm{AA}}=q_{\mathrm{BB}}=0.4$, (c) $q_{\mathrm{AB}}=q_{\mathrm{BA}}=1$ and $q_{\mathrm{AA}}=q_{\mathrm{BB}}=0.2$.

approach, from definition formula of the magnetic susceptibility, in accordance with the change of the applied magnetic fieldethod. $m_{\mathrm{A}}(\beta, h)$ and $m_{\mathrm{B}}(\beta, h)$ differential equation becomes each as follows: $m_{\mathrm{A}}(\beta, h+\Delta h)=$ $m_{\mathrm{A}}(\beta, h)+\chi_{\mathrm{A}}(\beta, h) \Delta h, \quad m_{\mathrm{B}}(\beta, h+\Delta h)=m_{\mathrm{B}}(\beta, h)+$ $\chi_{\mathrm{B}}(\beta, h) \Delta h$, where $m_{\mathrm{A}}=S^{\mathrm{A}} B_{\mathrm{S}^{\mathrm{A}}}\left(\beta \bar{h}_{\text {eff }}{ }^{\mathrm{A}}\right)$ and $m_{\mathrm{B}}=$ $S^{\mathrm{B}} B_{\mathrm{S}^{\mathrm{B}}}\left(\beta \bar{h}_{\mathrm{eff}}^{\mathrm{B}}\right)$ are given as minimum of free energy $\partial g / \partial m_{\mathrm{A}}=0$ and $\partial g / \partial m_{\mathrm{B}}=0$ respectively, $\chi_{\mathrm{A}}(\beta, h)$ and $\chi_{\mathrm{B}}(\beta, h)$ are magnetic susceptibilities in $\mathrm{A}$ and $\mathrm{B}$ site respectively. In the second approach, when varying the applied magnetic field according to the time change, from eq. (2), we derive the van Hove's phenomenological dynamical theory ${ }^{8)}$ as follows:

$$
\begin{aligned}
& m_{\mathrm{A}}(\beta, h(t)+\Delta h(t))=m_{\mathrm{A}}(\beta, h(t))-\left(\partial g / \partial m_{\mathrm{A}}\right)\left(\Delta t / \tau_{\mathrm{A}}\right), \\
& m_{\mathrm{A}}(\beta, h(t)+\Delta h(t))=m_{\mathrm{A}}(\beta, h(t))-\left(\partial g / \partial m_{\mathrm{A}}\right)\left(\Delta t / \tau_{\mathrm{A}}\right),
\end{aligned}
$$

where $\tau_{\mathrm{A}}$ and $\tau_{\mathrm{B}}$ are magnetic relaxation constants in $\mathrm{A}$ and $\mathrm{B}$ site respectively. At both first and second approach, in sufficiently low temperature than the critical temperature $T_{\mathrm{C}}$, the second term on the right side of these relationship to become very small, these are ignored in the first approximation. Thus, in our numerical simulations, it is represented using self consistent difference equations as follows:

$$
\begin{aligned}
& m_{\mathrm{A}}[\beta, h(t+\Delta t)] \simeq S^{\mathrm{A}} B_{\mathrm{S}^{\mathrm{A}}}\left(\beta \left\{q_{\mathrm{A}} m_{\mathrm{A}}[\beta, h(t)]\right.\right. \\
& \left.\left.\quad+q_{\mathrm{AB}} \varepsilon m_{\mathrm{B}}[\beta, h(t)]+h(t)\right\}\right), \\
& \varepsilon m_{\mathrm{B}}[\beta, h(t+\Delta t)] \simeq S^{\mathrm{B}} B_{\mathrm{S}^{\mathrm{B}}}\left(\beta \left\{q_{\mathrm{B}} \varepsilon m_{\mathrm{B}}[\beta, h(t)]\right.\right. \\
& \left.\left.\quad+q_{\mathrm{BA}} m_{\mathrm{A}}[\beta, h(t)]+h(t)\right\}\right),
\end{aligned}
$$

here, these symbols are defined by $q_{\mathrm{A}} \equiv z_{\mathrm{A}} J_{\mathrm{AA}}, q_{\mathrm{AB}} \equiv$ $-z_{\mathrm{AB}} J_{\mathrm{AB}}, q_{\mathrm{B}} \equiv z_{\mathrm{B}} J_{\mathrm{BB}}, q_{\mathrm{BA}} \equiv-z_{\mathrm{BA}} J_{\mathrm{BA}}$ and $\varepsilon \equiv \pm 1$. Time dependence of the applied magnetic field also is given by $h(t+\Delta t) \simeq h(t)+\Delta h$, and its difference width $\Delta h$ of the applied magnetic field is involve time interval $\Delta t$, it is related to the coercivity which is a parameter to be determined from the experimental results. Here we were provisionally estimated as about $\Delta h=63.3[\mathrm{Oe}]$. This may be considered to be associated with the Barkhausen jumps. ${ }^{9-14)}$ At Fig. 2(a), (b), (c) and (d), we shown the various parameters dependent 

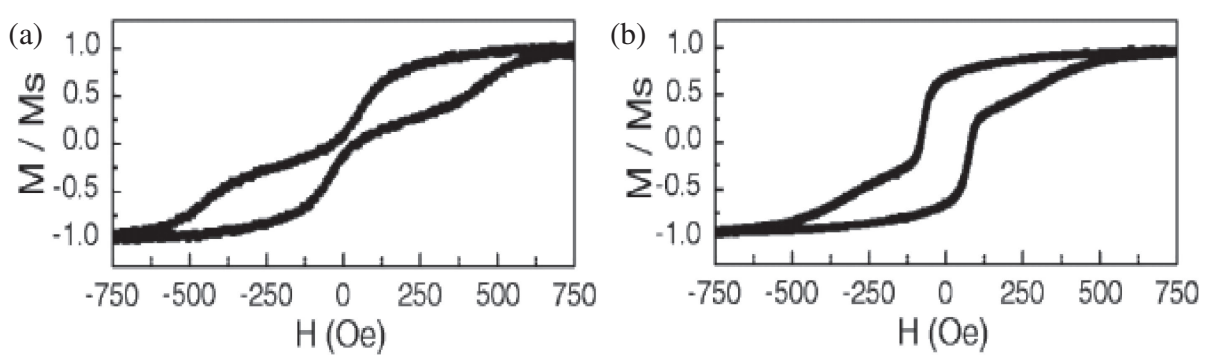

Fig. 3 This figure is a quote from the experimental results of Ref. 15). It is hysteresis loops of $\mathrm{Fe}_{3} \mathrm{O}_{4} / \mathrm{MgO} / \mathrm{Fe}_{3} \mathrm{O}_{4} / \mathrm{NiO}$ multilayers measured at $300[\mathrm{~K}]$ for different $\mathrm{MgO}$ thickness. The thickness of $\mathrm{MgO}$ spacer are (a) 0.8 [nm], and (b) 1.5 [nm], respectively.

magnetic hysteresis loops of $\mathrm{Fe}_{3} \mathrm{O}_{4}$ in temperature $T=300$ [K].

We make a comparison between the our simulation results and some experimental results. In Fig. 2(c) and (d), the shape of the hysteresis curve in the $\mathrm{Fe}_{3} \mathrm{O}_{4}$ that are submitted, show that it is a double loop. In Fig. 3, it shows the experimental results $^{15)}$ which are hysteresis loops for samples with different $\mathrm{MgO}$ thickness. The thickness of $\mathrm{MgO}$ spacer are $0.8[\mathrm{~nm}]$ and $1.5[\mathrm{~nm}]$ respectively. Also the experiments results in the Fig. 3(a) and (b), there are shown a sign of the double loop. Our simulation results, the cause of occurrence of the double loop characteristics of the hysteresis loop of the spin valve, indicating that it is the magnetic properties of the ferrimagnetic material.

\section{Summary and Discussion}

Summary of this paper is shown as follow. We have developed a simulation method of using a hysteresis curve of the ferrimagnetic material (mainly $\mathrm{Fe}_{3} \mathrm{O}_{4}$ ) by the retarded trace method. In particular, we revealed the physical meaning by retarded trace method from Hove's phenomenological dynamical theory. Our simulation results, the cause of occurrence of the double loop characteristics of the hysteresis loop of the spin valve, indicated that it is the magnetic properties of the ferrimagnetic material.

\section{Acknowledgments}

We are grateful to Professor Mitsuya Motohashi for helpful discussions.

\section{REFERENCES}

1) D. Tripathy, A. O. Adeyeye and S. Shannigrahi: Phys. Rev. B 75 (2007) 012403.

2) T. Kado: Appl. Phys. Lett. 92 (2008) 092502.

3) H.-C. Wu, O. N. Mryasov, M. Abid, K. Radican and I. V. Shvets: Scientific Reports 3 (2013) 1830.

4) S. Obata: Mater. Trans. 54 (2013) 1661-1677.

5) S. Obata: IEEJ Trans. FM 131 (2011) 838-845 (in Japanese).

6) S. Obata: J. Magn. Soc. Jpn. 36 (2012) 161-168 (in Japanese).

7) S. Obata: IEEJ Trans. FM 133 (2013) 489-499 (in Japanese).

8) L. Van Hove: Phys. Rev. 93 (1954) 1374.

9) S. Zapperi, P. Cizeau, G. Durin and E. Stanley: Phys. Rev. B 58 (1998) 6353-6366.

10) F. Colaiori and A. Moro: Adv. Phys. 57 (2008) 287-359.

11) S. Yang and J. L. Erskine: Phys. Rev. B 72 (2005) 064433.

12) K. Kova'cs and Z. Ne'da: J. Opt. Elect. Adv. Mater. 8 (2006) 10931097.

13) D. A. Christian, K. S. Novoselov and A. K. Geim: Phys. Rev. B 74 (2006) 06443.

14) G. Durin and S. Zapperi: J. Stat. Mech. 2006 (2006) P01002.

15) H.-C. Wu, S. K. Arora, O. N. Mryasov and I. V. Shvets: Appl. Phys. Lett. 92 (2008) 182502. 